\title{
ILCEA
}

Revue de l'Institut des langues et cultures

d'Europe, Amérique, Afrique, Asie et Australie

$15 \mid 2012$

Les mots de la crise

\section{Les mots de la crise : exploration du versant langagier de la crise boursière et financière de 2007-2009}

\section{(éditorial)}

The specialised lexicon of the 2007-2009 financial crisis: lexical peaks in a downhill economy

\section{Christian Leblond}

\section{OpenEdition}

\section{Journals}

Édition électronique

URL : http://journals.openedition.org/ilcea/1130

DOI : 10.4000/ilcea.1130

ISSN : 2101-0609

\section{Éditeur}

UGA Éditions/Université Grenoble Alpes

\section{Édition imprimée}

ISBN : 978-2-84310-220-2

ISSN : $1639-6073$

Référence électronique

Christian Leblond, «Les mots de la crise : exploration du versant langagier de la crise boursière et financière de 2007-2009 », ILCEA [En ligne], 15 | 2012, mis en ligne le 30 janvier 2012, consulté le 23 septembre 2020. URL : http://journals.openedition.org/ilcea/1130 ; DOI : https://doi.org/10.4000/ ilcea. 1130

Ce document a été généré automatiquement le 23 septembre 2020.

(C) ILCEA 


\title{
Les mots de la crise : exploration du versant langagier de la crise boursière et financière de 2007-2009
} (éditorial)

\author{
The specialised lexicon of the 2007-2009 financial crisis: lexical peaks in a \\ downhill economy
}

Christian Leblond

1 La crise économique que nous traversons dure depuis 2007, ce qui a donné amplement le temps aux observateurs de décrire le phénomène, aux spécialistes de le relier aux modèles théoriques et aux experts de formuler des solutions. Les travaux présentés ici n'ont pas pour ambition de trancher les multiples débats suscités par les soubresauts de l'économie, mais d'aborder la question des «mots de la crise» dans leur dimension traductologique et multilingue ${ }^{1}$. Les linguistes portent sur la crise économique un regard sinon tout à fait extérieur, du moins décentré et, nous l'espérons, relativement novateur. L'objectif du recueil est de décrire le déroulement des événements de la première phase de la crise à partir d'une étude statistique mesurant la fréquence d'emploi de certains termes dans la presse des pays d'Europe et d'Amérique du Nord pendant les mois concernés.

2 La question de la période d'étude, et de son autonomie par rapport au déroulement d'une dépression de longue durée se pose immédiatement. Le premier article traite, en ouverture, du cadrage chronologique définissant la "crise " comme la séquence identifiable d'un cycle. Cette définition objective est rudimentaire et sans doute provisoire, mais elle est opérationnelle et le manque de recul sur la période nous oblige à renvoyer à plus tard les implications épistémologiques de cette crise économique, qui débouchera vraisemblablement sur une crise de la représentation, avec l'éventuel changement de paradigme qui est à la clé.

3 En se basant sur les indices boursiers, la crise s'ouvre dès 2007 et atteint son point le plus sombre à l'automne 2008 avec la faillite, le 15 septembre 2008, de la grande banque 
Lehman Brothers, pourtant réputée too big to fail ${ }^{2}$. Le grand décrochage est parfaitement visible dans les derniers mois de 2008 et les minima sont atteints en mars 2009, point à partir duquel l'indice se redresse. Un bilan provisoire sera alors émis, l'assureur AIG (American Insurance Group) sera sauvé et la crise bancaire déclarée officiellement sous contrôle.

Il se dégage donc une certaine cohérence de la période, qui présente un "récit» complet sur trois années : 2007, 2008 et 2009, illustrant apparemment le retour aux remèdes keynésiens. Dans la séquence suivante en revanche, la crise des dettes souveraines semble orienter le scénario vers d'autres directions, et rien ne prouve que le consensus keynésien, un moment réhabilité après trois décennies de dérégulation, émerge triomphant. Cette partie de la crise est en vraie grandeur un test de la mondialisation des économies et des esprits. La crise se diffuse en Europe ${ }^{3}$ par des phénomènes à la fois financiers et psychologiques inextricablement mêlés selon le mécanisme des prédictions auto-réalisatrices qui donnent au langage un caractère performatif. L'inquiétude, puis la panique s'installe en même temps que certains mots terrifiants s'imposent à la une des journaux. Ces phénomènes psychologiques et leurs prolongements lexicaux induisent des effets réels quand les opérateurs en vue passent des ordres de vente massifs qui orientent les indicateurs à la baisse, et que ce signal amplifié déclenche l'effondrement des cours. Le langage ne se réduit pas à de lointains échos d'un drame qui se déroule dans le champ clos de l'économie, car l'émergence et la diffusion du lexique de la crise est partie prenante du phénomène. La panique s'exprime par des mots qui seront imprimés dans la presse spécialisée, repris dans les conversations, et lorsque le mot devient leitmotiv, que tous les journaux titrent sur l'éclatement de la bulle des prêts subprime, alors il n'est plus pertinent de distinguer la mimesis de l'écholalie, et celui qui déclare «c'est la crise » n'est pas différent de celui qui fait advenir la crise.

5 En 1933, dans un des plus célèbres discours de l'histoire des États-Unis, le président F. D. Roosevelt affirmait à ces concitoyens « la seule chose dont nous devons avoir peur est la peur elle-même $\mathrm{e}^{4}$ ». La crise comme la peur, est à la fois un phénomène subjectif et une réalité psychologique produisant un effet objectivement mesurable. Si la peur est la crise, en grande partie, les «mots de la crise » sont également la crise. Cette paroleaction, cette fusion détonante de l'action et de l'énonciation, va balayer l'édifice qui tenait en équilibre par le jeu subtil des « anticipations rationnelles».

6 Les manifestations langagières accompagnent et parfois précèdent les évènements de nature strictement économiques et quantifiables en tant que tel - comme l'évolution des indices. C'est pourquoi le premier axe suggéré aux participants est un repérage chronologique précis pour établir éventuellement un schéma de corrélation, voire un modèle prédictif. Le point de départ géographique de la crise étant aux États-Unis, c'est à partir des termes employés dans ce pays pour désigner les phénomènes (par exemple subprime), que l'on cherchera à mesurer la rapidité de la propagation de la crise en étudiant les décalages temporels d'un pays à l'autre. La méthodologie privilégiée pour obtenir des éléments de réponse à cette problématique chronologique est le recensement des pics d'usage dans les corpus paramétrés selon des critères comparables dans les différents pays européens.

7 La deuxième piste envisagée par l'équipe est d'adopter une problématique de traductologie proprement dite, en se posant tout particulièrement la question de la non-traduction de certains éléments lexicaux de l'anglais qui demeurent des emprunts 
tout à fait repérables. Des problématiques dérivant des préoccupations de traductologie peuvent se rattacher à ce thème, par exemple en distinguant le cas de la presse généraliste ou spécialisée, qui peut traduire ou conserver l'emprunt sous sa forme originale pour des raisons différentes.

Enfin une troisième dimension exploratoire du phénomène est envisageable, en privilégiant l'examen des prolongements métaphoriques et phraséologiques des termes spécialisés dans les différentes langues.

9 Les fluctuations du cycle économique, dont on perçoit a posteriori la régularité presque rassurante, confère une importance particulière, sur fond de phénomène cyclique, à l'inédit, aux éléments distinctifs qui vont jouer le rôle de marqueurs, et parmi eux, par définition, les néologismes qui constituent les «mots de la crise». Du strict point de vue économique, l'éternel retour des conjonctures hautes et basses réserve toujours une part à cet inédit. D'ailleurs, la crise de 2007-2009 se distingue fortement des précédentes car elle n'est pas de type stop and go caractéristique de l'après-guerre. Elle a beaucoup de points communs avec celle des années 1930 qui était une crise de surinvestissement ${ }^{5}$. Pour cela deux facteurs sont présents en amont: une épargne abondante y compris hors des États-Unis, et de faibles taux d'intérêt qui incitent à emprunter pour investir ${ }^{6}$, dopant la cote des valeurs mobilières et de l'immobilier?

10 L'envolée des cours, et le volume des transactions crée une bulle, phénomène complexe, qui ne se résout pas par des ajustements progressifs de type dialectique mais par de brusques retournements, selon une typologie décrite par Bordron (2011), à la suite de Saussure :

L'articulation du temps, telle que Saussure semble la comprendre dans le Cours, induit une logique de l'histoire dans laquelle les actions seraient absolument aveugles à ce qu'elles produisent selon le principe, déjà cité, qui nous dit : «[...] un fait diachronique est un événement qui a sa raison d'être en lui-même; les conséquences synchroniques particulières qui peuvent en découler lui sont complètement étrangères. » La dualité des domaines de valeurs ne débouche pas sur une dialectique transformatrice, toujours plus ou moins téléologique, mais sur des faits d'émergence, de réorganisation, de rupture, dont la caractéristique première est d'être imprévisible.

11 Les phénomènes «d'émergence, de réorganisation, de rupture» que décrit la linguistique accompagnent effectivement la dernière phase d'une bulle, ce qui explique le rôle de premier plan qu'occupent alors les personnages de type oraculaire - au premier rang desquels le gouverneur de la $\mathrm{FED}^{8}$ mais quelquefois aussi des personnages moins recommandables qui nous conduisent aux confins de la pensée magique. Le mauvais augure n'est plus l'envol des oiseaux vers la gauche, mais un " point de base ${ }^{9}$ " en plus ou en moins aux taux d'intérêt, accompagné d'une déclaration sibylline du grand initié dont un second cercle décode, à son tour, les vaticinations.

Il faut rappeler que les actifs financiers ne suivent pas la loi classique de l'offre et de la demande, qui aboutit à des variations de faible amplitude autour d'un point d'équilibre. En faisant intervenir des concepts tels que l'élasticité, il est possible de rendre compte des évolutions de la demande d'un produit par rapport au prix ou au revenu par exemple. Pour les actifs financiers, la hausse des cours ne provoque pas nécessairement une baisse de la demande, comme pour un bien ordinaire, mais peut être décodée comme la promesse de plus-value, et donc inciter au contraire les investisseurs à gonfler leur portefeuille de valeurs. Plus l'information migre des « experts » vers les "profanes", plus se répand la conscience que le moment de l'ajustement brutal se 
rapproche, ce que l'étude statistique de la diffusion des mots de la crise dans le temps et l'espace permet sans doute - fort modestement - de modéliser.

En matière d'information économique, le lecteur de la presse est au minimum un spectateur engagé. Comme il est quasiment impossible de vivre dans nos sociétés modernes sans effectuer des choix économiques, ne serait-ce qu'en consommant ou en exerçant notre "préférence pour la liquidité ${ }^{10}$ ", les lecteurs sont presque tous des acteurs. L'information économique à laquelle nous avons accès conditionne les décisions individuelles, et façonne la réalité collective à des degrés divers. En effet, si certains font de la figuration, comme les inactifs ou les petits porteurs, dans la démocratie censitaire que constitue le marché, les « décideurs » occupent le devant de la scène. Aux commandes de l'économie, dans le cercle fermé des conseils d'administration ou du pouvoir politique, ils ont accès à des informations exclusives, ou s'ils sont placés sur un échelon moins élevé de la hiérarchie économique ou administrative, ils ont les moyens de s'abonner à des newsletters dont le tarif élevé semble confirmer le caractère confidentiel.

Le commun des mortels lit la presse ou consulte les sites en ligne, qui diffusent en différé les informations hier confidentielles et aujourd'hui sans doute démonétisées parce que banales. Dans cette version du meilleur des mondes possibles, même le candide finit par avoir accès à l'information, mais en bout de chaîne. Le décryptage progressif d'une réalité complexe s'achève - telle est notre thèse-par une simplification radicale et la diffusion quasiment universelle d'une série de mots-clés.

Ceux-ci ne sont pas en soi performatifs, mais il est probable qu'un saut qualitatif se produit à partir d'un certain degré d'accumulation des occurrences de ces termes dans les échanges et dans la presse. On peut penser que ce caractère mimétique (la périphérie imite le centre) est une caractéristique des marchés boursiers, dont le caractère moutonnier a été magistralement décrit au moment de la Grande Crise des années trente par J. M. Keynes (1935) :

It might have been supposed that competition between expert professionals, possessing judgment and knowledge beyond that of the average private investor, would correct the vagaries of the ignorant individual left to himself. It happens, however, that the energies and skill of the professional investor and speculator are mainly occupied otherwise. For most of these persons are, in fact, largely concerned, not with making superior long-term forecasts of the probable yield of an investment over its whole life, but with foreseeing changes in the conventional basis of valuation a short time ahead of the general public. They are concerned, not with what an investment is really worth to a man who buys it 'for keeps', but with what the market will value it at, under the influence of mass psychology, three months or a year hence. Moreover, this behaviour is not the outcome of a wrongheaded propensity. It is an inevitable result of an investment market organised along the lines described. [...] Thus the professional investor is forced to concern himself with the anticipation of impending changes, in the news or in the atmosphere, of the kind by which experience shows that the mass psychology of the market is most influenced.

Le concept développé ici met à jour des boucles de rétroactions, où réalité et conscience de la réalité interagissent, où l'observateur transforme ce qu'il aperçoit, et où les mots se chargent d'un pouvoir redoutable. Mais des métaphores plus légères, sans pour autant manquer de profondeur, sont souvent proposées, depuis les «chaises musicales » jusqu'au célèbre « concours de beauté11 ${ }^{\text {». }}$ 
17 Le projet présenté dans ce recueil partira d'une liste volontairement restreinte de «mots de la crise», en posant l'hypothèse que le resserrement du lexique - le phénomène du sablier - focalise les angoisses. Un lexique de plus en plus étroit, et l'accélération des occurrences finit par donner aux mots de la crise une sorte d'omniprésence, ce qui nous porte à penser que la violence de la déflagration est inversement proportionnelle à la variété du lexique de la crise - et nous incite à rechercher le mot ultime qui joue le rôle de la particule élémentaire étymologiquement l'atome.

18 Si l'on médite l'analyse de Frédéric Hanin (2006), il est possible de rattacher la démarche du lexicologue qui s'attache à la spécificité d'une production langagière à certaines approches d'économistes :

La méthodologie cambridgienne développée par Marshall, dont Keynes hérite en partie, va mettre l'accent sur les situations locales, que ce soit dans l'espace ou dans le temps. S'il existe des lois économiques, elles sont contingentes à une période historique donnée [...]. Dans cette perspective, il s'agit moins de fonder l'analyse économique sur des caractéristiques communes aux différentes périodes historiques, que de rechercher ce qui est spécifique à la période que l'on cherche à étudier.

19 En effet, nommer à l'unanimité la crise d'un seul mot serait le moment paroxystique et évidemment impossible d'un drame collectif ${ }^{12}$ qui prendrait les traits d'une mimesis simplificatrice, peut-être une mimesis d'appropriation au sens de Girard ${ }^{13}$. Ce moment déboucherait sur une catharsis et, plus prosaïquement, sur la seconde partie du cycle économique, la reprise des affaires, ce qui est, après tout, une fonction de la crise.

La mise en récit archétypal au nom de la rationalité individuelle conduit à la négation de la diversité qui est aussi une condition de l'équilibre des marchés ${ }^{14}$. C'est ainsi que le projet s'est structuré autour d'une liste de termes anglais (voir article de C. Leblond) qui constitue le point d'arrivée d'une longue série de tests selon la méthode décrite à la fin de cette présentation, mais qui sert de point de départ à l'étude traductologique et phraséologique dans les autres langues. Après de nombreuses navettes, suivies de validation, les termes anglais ont été classés du plus général au plus spécifique. Partant $\mathrm{du}$ «bruit de fond» qui trahit l'inquiétude latente des cambistes sur une période longue, en passant par l'analyse (nécessairement rapide) des modes d'organisation typiquement anglo-saxons qui compliquent la tâche du traducteur, la liste finit sur des termes de plus en plus spécifiques, qui constituent le noyau dur des « mots de la crise ».

21 Pour la langue anglaise, le lecteur trouvera dans l'article de Christian Leblond des éléments de cadrage, méthodologico-chronologiques, puis le lexique commenté qui a été adopté par l'équipe comme dispositif commun de départ, avant de passer, à travers l'étude des occurrences, à une analyse des enjeux et périls de la traduction - et de la non-traduction. Toujours dans le domaine américain, Rudi Palmieri, à qui nous devons le second article, opte pour une focalisation précise sur la crise du secteur automobile et s'attache à mettre en lumière l'appareil argumentatif qui sous-tend la communication des grands constructeurs de Detroit dans un contexte de quasi-faillite.

Pour la langue allemande, l'article signé par Claire Allignol et Élodie Vargas, s'attache à exploiter l'outil Factiva pour détailler, au-delà de l'étude statistique, les reformulations et les métaphores de la crise attestées dans la presse germanophone. Ce prolongement phraséologique et stylistique donne un aperçu saisissant de la mission du « journaliste vulgarisateur » et des contraintes auxquelles il est soumis. 


$$
\begin{aligned}
& \text { initialement - permet de générer des histogrammes illustrant le nombre d'occurrences } \\
& \text { d'un mot précis (sur l'ordonnée) pendant une période paramétrable et divisible (le plus } \\
& \text { souvent année divisée en mois sur l'abscisse). Ces histogrammes sont utilisables dans le } \\
& \text { cadre de l'étude de la corrélation temporelle entre les phénomènes économiques et }
\end{aligned}
$$

ILCEA, 15 | 2012 
lexicaux, mais aussi -ultérieurement - entre manifestations langagières dans différents pays. Notons enfin, que le paramétrage de Factiva, même s'il est moins sophistiqué que celui d'autres outils en ligne, permet d'affiner ou d'élargir les requêtes par l'usage de wildcards ${ }^{15}$, et le choix entre "phrase exacte » très utile pour mesurer la fréquence d'une expression figée, et «au moins un de ces mots » si deux termes sont à associer indifféremment pour la même requête ${ }^{16}$.

\section{BIBLIOGRAPHIE}

BORDRON Jean-François, « Trois ordres de la valeur selon la qualité, la quantité et la relation » [en ligne], Semen, $\mathrm{n}^{\circ} 32,2011$, mis en ligne le $1^{\mathrm{er}}$ octobre 2011. Disponible sur <http:// semen.revues.org/9344> [consulté le 6 décembre 2011].

GIRARD René, La violence et le Sacré, Hachette, 1972.

HANIN Frédéric, «Les dimensions de la sociologie économique de la finance : Perspective critique, transformations institutionnelles et facteurs collectifs » [en ligne], Revue Interventions économiques, $\mathrm{n}^{\mathrm{0}} 33,2006$, mis en ligne le $1^{\mathrm{er}}$ avril 2006. Disponible sur <http:// interventionseconomiques.revues.org/804> [consulté le 9 décembre 2011].

KEYNES John Maynard, The General Theory of Employment, Interest and Money, Cambridge University Press, 1936 [rédigé en 1935]. Disponible sur <http://www.marxists.org/reference/subject/ economics/keynes/general-theory/>

MISHKIN Frederic et al., Monnaie, banque et marchés financiers,Pearson, 2010.

\section{NOTES}

1. Le projet de recherche a été lancé par une équipe constituée de membres du Groupe de Recherche Multilingue sur la Traduction, le GREMUTS, composante de l'ILCEA. C'est toute la production journalistique (presse spécialisée ou généraliste) et technique (experts, revues savantes) dans les langues européennes qui est envisagée comme pouvant fournir un corpus dans ce cadre.

2. C'est-à-dire de trop grande taille pour que les autorités monétaires (la FED) et le ministère des finances (Treasury) laissent advenir une faillite, qui présenterait un risque systémique. Les évènements vont démontrer le contraire.

3. F. Mishkin explique (p. 425) comment une première banque allemande IKB Deutsche Industrie Bank essuie des pertes massives en juillet 2007.

4. Discours d'investiture de F. D. Roosevelt en 1933 consultable sur < http://www.archives.gov/ education/lessons/fdr-inaugural/>

5. En anglais américain, overinvestment est souvent remplacé par glut, même sous la plume des économistes et des banquiers, terme qui évoque la satiété, voire le péché de gourmandise.

6. En jouant sur les effets « levier ». Le levier augmente l'amplitude des variations à la hausse, mais aussi à la baisse. Les pertes en capital, quand les positions le dénouent, peuvent excéder la mise de fonds initiale. 
7. Par convention, les ménages qui achètent un bien immobilier ne consomment pas, ils investissent.

8. Le mélange de technicité, d'autorité auréolée de mystère dessinent le portrait d'un initié versé dans l'alchimie boursière à qui l'on peut confier la gestion de son portefeuille sans vraiment comprendre le mécanisme qui permet de servir des rémunérations bien supérieures à la moyenne, d'où le succès de Bernard Madoff et de son lointain prédécesseur Charles Ponzi.

9. C'est-à-dire un dixième de point de pourcentage.

10. Le concept est keynésien (un investisseur choisit les formes de placement les plus liquides quand il est inquiet pour l'avenir).

11. Concours où, comme l'explique malicieusement J. M. Keynes, chaque membre du jury, au lieu de voter simplement pour la candidate qu'il trouve la plus jolie, essaie de deviner laquelle aura le plus de succès aux yeux des autres - afin de passer personnellement pour un homme de goût.

12. La crise est le moment où tout le monde énonce l'opinion suivante: "c'est la crise". Nommer une bulle (« la bulle des subprimes »), c'est de fait provoquer son éclatement. D'ailleurs une plaisanterie d'économiste assez fréquente consiste à dire que l'on reconnaît une bulle quand elle éclate, et jamais avant.

13. Voir notamment les pages sur la pathologie cyclothymique (p. 229 et suiv.).

14. L'équilibre est certes dynamique avec des cambistes qui changent leurs positions et font évoluer leur portefeuille. Une des règles prudentielles étant la diversification des types d'actifs, quand tous les agents économiques vont au bout de la logique mimétique (tous haussiers puis tous baissiers) - et qu'ils adoptent ce faisant le même langage - le système devient extrêmement instable. La métaphore du bateau qui chavire parce que tous les passagers se portent du même côté fournit une bonne illustration.

15. Par exemple repos* est une requête qui regroupe le verbe repossess et le substantif repossession.

16. Pour ne pas à comptabiliser séparément la fréquence de termes correspondant au même phénomène comme foreclosure et repossession.

\section{AUTEUR}

\section{CHRISTIAN LEBLOND}

MCF, Université Stendhal - Grenoble 3. ILCEA 